\title{
False high level in total bilirubin estimation in nonicteric serum
}

\author{
Saha BISWAJIT \\ Department of Biochemistry, Durgapur Steel Plant Hospital, Durgapur -713205, West Bengal, India. \\ E-mail:biswajitsahadsp@gmail.com; Tel: +91 9434792405
}

\begin{abstract}
In day to day clinical biochemistry laboratory practices, occasionally abnormal levels of individual parameters are noted. These reports cannot be explained immediately with certainty always. Inquisitiveness with in-depth analysis might reveal the possible cause sometimes. To find out the possible cause of false elevation in total bilirubin estimation in nonicteric serum from one patient was the objective. Routine estimation of total bilirubin in serum/plasma by the decade old practice of using very reliable and stable reagent kit from Roche in the biochemistry laboratory of a general hospital revealed a falsely very high value of total bilirubin in a patient's blood sample for the first time. Subsequently, the same sample was subjected to estimation of total bilirubin by DiaSys and Randox reagents along with simultaneous re-estimation by Roche reagents in next three days. Repeated estimations by Roche reagents showed falsely very high level of total bilirubin. However, estimation by DiaSys and Randox reagents showed acceptable normal levels as per visual estimation. There was interference, most probably due to paraprotein in the estimation of total bilirubin by Roche reagents but not by DiaSys and Randox reagents in that particular sample.
\end{abstract}

(C) 2017 International Formulae Group. All rights reserved.

Keywords: False, high, positive, total bilirubin, nonicteric, serum.

\section{INTRODUCTION}

Total bilirubin estimation has been done by automated clinical chemistry analyzer popularly known as auto analyzer using commercially available quality reagent kits either made by the original equipment manufacturer ("closed" system) or by other company's reagent kits as well if the auto analyzer is so designed ("open" system).

As a regular practice, any clinical biochemistry laboratory of a hospital or diagnostic center uses only one reagent from a particular make/company after ensuring its quality performance for day to day routine biochemical investigations from blood for patients' care services for a specified parameter over a period of time depending on the situation primarily due to costeffectiveness.

Olympus AU 640 auto analyzer (later named Beckman Coulter) is an "open" system machine and total bilirubin reagent kit from Roche (Reagent literature of Roche total bilirubin) has been used by our 600 bed hospital for ten years without any analytical problem. Earlier, over a period of considerable period, estimation of some random samples of varying concentrations of total bilirubin and direct bilirubin using Beckman Coulter reagents (Reagent literature 
of Beckman Coulter total bilirubin, direct bilirubin) showed good correlation with Roche make reagents (Reagent literature of Roche total bilirubin, direct bilirubin) with but showed stability for much longer period and better quality performance. Hence, the choice. Later direct bilirubin from DiaSys (Reagent literature of Diasys direct bilirubin) showed good correlation with Roche reagents (Reagent literature of Roche direct bilirubin) with almost equal stability and quality performance. DiaSys make direct bilirubin has been used mainly due to slightly lower cost in a public sector hospital. Subsequently, total bilirubin estimation over a considerable period using DiaSys and Randox reagents (Reagent literature of DiaSys and Randox total bilirubin) in the above auto analyzers showed comparable results with Roche reagents. Hence, technically, reagents from all the above companies were acceptable and in fact, have been used by Olympus AU series auto analyzers at different places. The limits of interferences from haemolysis and lipemia both in total and direct bilirubin assay in the reagent literature of all the above three companies had been indicated. In addition, information regarding interference in total bilirubin estimation by indican, cyclosporine, hydroxycobalamin and in some multiple myeloma patients with varying degree of positive bias in Roche, by monoclonal IgM (Waldenstorm's macroglobulinaemia) rarely in Beckman Coulter and by ascorbic acid in DiaSys make reagents were available. Similarly, interference in direct bilirubin estimation by phenylbutazone in Roche and ascorbic acid in DiaSys had been mentioned. Rarely, monoclonal IgM (Waldenstorm's macroglobulinaemia) could interfere in direct bilirubin estimation both in Roche and Beckman Coulter reagents.

Recently, a blood sample showed a false positive interference to a significant extent in total bilirubin estimation by Roche reagent, the probable cause of which needed to be looked into.

\section{MATERIALS AND METHODS}

Liver function tests (LFT) were done in a blood sample received from a 62 years male patient admitted in a medical ward. Conventionally, direct bilirubin estimation was not done by our laboratory unless specifically asked for the same. After observing a strikingly very high level of total bilirubin in a non-icteric (almost watery) serum along with slightly high level of total protein, low level of albumin, reversal of albumin globulin ratio, marginally elevated normal alkaline phosphatase and normal alanine aminotransferase even on repeat estimation, a fresh sample was sought on emergency basis primarily to rule out any contamination. The difference of sampling time between the two samples was about five hours. The physical appearance of both the samples was same and the results were also similar.

The centrifuged sample was kept in the primary tube along with the blood cells in the air-conditioned laboratory overnight. Next morning, the sample was subjected to reestimation as a routine sample for all the parameters of LFT in addition to direct bilirubin by Diasys reagents (Rand and Di Pasqua,1962) which had been in use in the laboratory. The serum was separated from the sample and the same was kept in the freezing chamber of the refrigerator. Next day, analysis with a Diasys make total bilirubin (Rand and Di Pasqua, 1962) was done along with Roche make total bilirubin (Wahlefeld et al., 1972). Next day, i.e. on the $4^{\text {th }}$ day, analysis with a Randox make total bilirubin (Jendrassik and Grof, 1938) was also done along with both Roche and DiaSys make reagent.

\section{RESULTS AND DISCUSSION}

Although the actual level of total bilirubin appeared to be around $0.3 \mathrm{mg} / \mathrm{dl}$ as per visual estimation, the results in Table 1 showed that the level of total bilirubin (mean $7.6 \mathrm{mg} / \mathrm{dl}$ ) only measured using Roche 
reagents was found repeatedly considerably higher than that of total bilirubin using DiaSys $(0.2 \mathrm{mg} / \mathrm{dl})$ and Randox $(0.4 \mathrm{mg} / \mathrm{dl})$ reagents by 38 times $(0.2 \mathrm{mg} / \mathrm{dl})$ and 19 times $((0.4$ $\mathrm{mg} / \mathrm{dl})$ respectively. The latter two corresponded well to visual estimation. This apparently remarkable difference between DiaSys / Randox and Roche did not have any clinical significance. However, the level measured by Roche reagents was not only unacceptable but also sufficient to create confusion. The patient after being symptomfree was waiting to be released. After interacting with the treating doctor personally and then appraising of the false positive high result, the patient was discharged shortly after receipt of the above report as per his wish.

In order to confirm or refute the possibility of the same obvious technical interference in total bilirubin estimation by other reliable sources, this particular investigation was carried out using DiaSys and Randox reagents. The delay of four days for subsequent estimations was primarily due to immediate nonavailability of Dia Sys and Randox reagents at this geographical location.

After scrutiny of the case details from the hospital records, it was learnt that the LFT done on the preceding day showed the similar results (T.B. $-7.4 \mathrm{mg} / \mathrm{dl}$, T.P. $-10 \mathrm{~g} / \mathrm{dl}$, Alb.$2.4 \mathrm{~g} / \mathrm{dl}$, Glob. - 7.6 g/dl , A:G - 0.31, ALP $102 \mathrm{U} / \mathrm{L}$ and ALT $-15 \mathrm{U} / \mathrm{L}$ ) and the sample was essentially sent for reconfirmation of the reports of the patient who was admitted to our hospital five days back with bilateral upper back pain. On examination, tenderness in the thoracic 11 and 12 vertebrae were noted. The patient became symptom-free after symptomatic treatment and was discharged. Investigations showed there was no bony changes in the thoraco-lumbar X-ray imaging but significant haematological findings included haemoglobin $-5.9 \mathrm{mg} / \mathrm{dl}$, erythrocyte sedimentation rate $-165 \mathrm{~mm} \mathrm{(1{ } ^ { \text { st } }}$ hour) and white blood cell count $4,400 / \mathrm{cmm}$. All these were suggestive that the patient might be suffering probably from early multiple myeloma.

Review of literature further revealed that total and direct bilirubin assay were affected due to interference from naproxen, Odesmethylnaproxen (a metabolite of naproxen), indican and paraprotein by Roche, Beckman Coulter and Siemens machine/reagents (Garber, 1981; Miller et al., 2004; Riyami et al., 2009; Dasgupta et al., 2010). Artefactual hyperbilirubinaemia due to paraprotein interference in total bilirubin estimation but not direct bilirubin in two index patients (Pantanowitz et al, 2003), one patient with monoclonal IgM paraprotein (Smogorzewska et al., 2004) and one patient with high IgG level in multiple myeloma (Singh et al., 2015) using Roche reagents have been reported. Hence, even among the multiple myeloma patients, the incidence of this spurious elevation in total bilirubin was, indeed, rare. Observation of similar result in this case was in agreement with others.

It was of concern to note that the chemical name including its quantity of detergent/surfactant had not been mentioned in any reagent literature of total bilirubin estimation (Regent Literature of Roche, DiaSys and Randox). Keeping the above in view, the probable interference in the estimation of total bilirubin only in this case seemed to be the very rare possibility of paraprotein, the reason being the Roche solubilizing agent which failed to solubilize properly if the IgG levels probably exceeded about $2000-3000 \mathrm{mg} / \mathrm{dl}$ (Singh et al., 2015) or presumably due to its quantity or quality and seemingly, the same with other paraproteins too. This caused variable precipitation of protein depending on its concentration and isoelectric point at the acidic $\mathrm{pH}$ of 1-2 which resulted in increased absorbance instead of producing color proportionate to the actual concentration of total bilirubin. Encountering with the same problem would be anticipated with Beckman Coulter reagent also since 3,5- 
dichlorophenyldiazonium (DPD) had been used in that reagent kit. Hence, method using DPD had this rare drawback. However, this did not occur with the method that used diatotized 2,4-dichloroaniline although DPD was also used (DiaSys) or diazotized sulfanilic acid (Randox) including perhaps, the quality and quantity of solubilizing agent.

The main deficiencies in the above case were nonestimation of direct bilirubin both by Roche and Randox reagents and subsequent evaluation by serum electrophoresis including immunoelectrophoresis to confirm or rule out multiple myeloma since the patient did not turn up later for follow up. It might be pertinent to mention that non-availability of Roche make reagents due to withdrawal of its "open" reagents from the market precluded direct bilirubin estimation using Roche reagents. Procurement of multiple reagent kits for individual parameters and using those daily for estimation of this type of rare sample only in a public sector hospital was not practically possible. However, it needed to be appreciated that finding out the a correct explanation quickly for any parameter was not feasible in the resource-poor setting of a high output hospital clinical laboratory more than the way the above case was investigated.

Repeated evaluations by a particular reagent in any specified clinical laboratory and repeated cross-checking by another laboratory using a different reagent in the above type of case will continue to show gross discrepancy. Hence, it is concluded that unless one becomes very much careful during validation of report for this type of rare problem in a busy routine laboratory, it is almost certain to create great confusion particularly in the setting of absence of provision of interaction with the treating doctor regardless of the condition of the patient and no laboratory ought to be blamed prudently for the obvious inherent limitation of the reagents.

Table 1: The levels of different biochemical parameters of liver function tests.

\begin{tabular}{lcccccccccc}
\hline Sample & T.B. & D.B. & T.B.D. & T.B.R. & T.P. & Alb. & Glob. & A: G & ALP & ALT \\
Unit & $\mathrm{mg} / \mathrm{dl}$ & $\mathrm{mg} / \mathrm{dl}$ & $\mathrm{mg} / \mathrm{dl}$ & $\mathrm{mg} / \mathrm{dl}$ & $\mathrm{g} / \mathrm{dl}$ & $\mathrm{g} / \mathrm{dl}$ & $\mathrm{g} / \mathrm{dl}$ & & $\mathrm{U} / \mathrm{L}$ & $\mathrm{U} / \mathrm{L}$ \\
\hline A & 7.6 & & & & 10.3 & 2.5 & 7.8 & 0.32 & 128 & 18 \\
B & 7.4 & & & & 9.7 & 2.3 & 7.4 & 0.31 & 121 & 12 \\
C & 6.7 & 0.3 & & & 9.4 & 2.1 & 9.3 & 0.22 & 116 & 14 \\
D & 8.1 & 0.1 & 0.2 & & 9.8 & 2.2 & 7.6 & 0.28 & & \\
E & 8.4 & 0.1 & 0.2 & 0.4 & & & & & & \\
\hline
\end{tabular}

[ $\mathrm{A}=1^{\text {st }}$ sample of the $1^{\text {st }}$ day, $\mathrm{B}=2^{\text {nd }}$ sample of the $1^{\text {st }}$ day, C, D \& E $=\mathrm{B}$ estimated on the $3^{\text {rd }}, 4^{\text {th }} \& 5^{\text {th }}$ day $;$ T.B. $=$ Total Bilirubin (Roche,RI* : up to $1.2 \mathrm{mg} / \mathrm{dl}$ ), D.B. $=$ Direct Bilirubin (DiaSys, $\mathrm{RI}^{*}:<0.2 \mathrm{mg} / \mathrm{dl}$ ), TBD $=$ Total Bilirubin (DiaSys, $\mathrm{RI}^{*}$ : 0.1-1.2 mg/dl), TBR = Total Bilirubin (Randox, RI* : up to $1 \mathrm{mg} / \mathrm{dl}$ ) T.P. = Total Protein(Roche, RI* : 6-8 g/dl ), Alb.= Albumin $\left(\mathrm{BC}^{\#}, \mathrm{RI}-3.5-5.2 \mathrm{~g} / \mathrm{dl}\right) \mathrm{Glob}=$ Globulin $\left(\mathrm{RI}^{*}-1.5-2.5 \mathrm{mg} / \mathrm{dl}, \mathrm{A}: \mathrm{G}=\right.$ Albumin : Globulin ratio, RI-1.5:1, ALP = Alkaline phosphatase $\left(\mathrm{BC}^{\#}, \mathrm{RI}^{*}\right.$ - 30-120) $\mathrm{ALT}=$ Alanine aminotranferase $\left(\mathrm{BC}^{\#}, \mathrm{RI}^{*}-<50 \mathrm{U} / \mathrm{L}\right)\left({ }^{*} \mathrm{RI}=\right.$ Reference Interval, $\mathrm{BC}^{\#}=$ Beckman Coulter). 


\section{COMPETING INTERESTS}

The author declares that there is no competing interest.

\section{AUTHORS' CONTRIBUTIONS}

This is a single authored paper; the author conceived, carried out the study, wrote and typed the manuscript.

\section{ACKNOWLEDGEMENTS}

Both the local agency of DiaSys and Randox in Kolkata, West Bengal, India offered a box of reagent for estimation of total bilirubin on request free of cost and the same of Roche offered the literature of direct bilirubin estimation.

\section{REFERENCES}

Al Riyami N, Zimmermann AC, Rosenberg FM, Holmes DTl, 2009. Spurious hyperbilirubinaemia caused by naproxen. Clin. Biochem., 42 (1-2): 129-131. DOI: org/10.1016/j.clinbiochem.2008.09.119

Dasgupta A, Langmen LJ, Johnson M, Chow L. 2010. Naproxen metabolites interfere with certain bilirubin asays. Elimination of interference by using a Roche bilirubin assay on the Hitachi 917 analyzer. Am. J. Clin. Pathol., 133(6): 878-883.

DOI:

\subsection{9/AJCPN6MWATQ3SZTC}

Garber CC. 1981. Jendrassic-Grof analysis for total and direct bilirubin in serum with a centrifugal analyzer. Clin. Chem., 27(8): 1410-1416.

DOI: https://www.ncbi.nlm.nih.gov/pubmed/7 273400

Jendrassik L, Grof P. 1938. Vereinfachte photometrische methoden zur bestimmung des bilirubins. Biochem. Z., 297: 82-89.
Miller MA, Fortson S, Bose S. 2004. A false elevation of bilirubin in blood and urine of a naproxen overdose patient. $J$. Toxicol. Clin. Toxicol., 42: 793.

Pantanowitz L, Horowitz GL, Upalakin JN, Beckwith BA. 2003. Artefactual hyperbilirubinaemia due to paraprotein interference. Arch. Pathol. Lab. Med., 127(1): $\quad 55-59 . \quad$ DOI: https://www.ncbi.nlm.nih.go

v/pubmed/12521367

Reagent literature of total bilirubin (BILT3, Bilirubin Total Gen.3), Cat.No. 05795265, Roche/Cobas, Roche Diagnostics GmBH, SandhoferStrasse 116,D-68305 Mannheim. DOI: https://pim-eservicestst.roche.com/eLD/.../be/en/Documents/ GetDocument?..

Rand RN, diPasqua A. 1962. A new diazo method for the determination of bilirubin. Clin. Chem., 8: 570-578. DOI: https://www.ncbi.nlm.nih.gov/pubmed/1 3990737

Reagent literature of direct bilirubin [BILD2, Bilirubin Direct Gen.2 (Doumas standardization], Cat. No. 05589061, Roche/Cobas, Roche Diagnostics GmBH, Sandhofertraessean 116,D68305 Mannheim. DOI: www.rochecanada.com/.../roche.../05589 061190\%20BilBD2-C311Can\%20En\%20V...

Reagent literature of Bilirubin Auto Total FS, DiaSys, Cat. No - 108119010962, DiaSys Diagnostic Systems GmBH, Alte Strasse9 65558 Holzheim, Germany. DOI: www.diasysdiagnostics.com/products/kit.../kits.../pro duct...bilirubin-auto-total-fs/pro... 
Reagent literature of Bilrubin Auto Direct FS,

DiaSys, Cat. No. 108219910704,

DiaSys Diagnostic Systems GmBH, Alte

Strasse9 65558 Holzheim, Germany.

DOI: $\quad$ www.diasys-

diagnostics.com/products/kit...kits/produ

ct...bilirubin-auto-direct-fs/prod...

Reagent literature of Total Bilirubin (T Bil),

Rx Series BR 3859, Randox, Randox

Laboratories Ltd, 55 Diamond Road,

Crumlin, County Antrim, BT29 4QY,

United Kingdom.

Reagent literature of Total Bilirubin,

Beckman Coulter (OSR 6112/6212),

Beckman Coulter Inc.,2505, Kraemer

Blvd. Brea, C 92821, USA. DOI:

https://www.beckmancoulter.com/.../tech

docs?.../BAOSR6x 12/...BILIRUBIN_TO

TAL

Reagent literature of Direct Bilirubin,

Beckman Coulter (OSR 6111/6211),

Beckman Coulter Inc.,2505, Kraemer
Blvd. Brea, C 92821, USA. DOI: https://www.beckmancoulter.com/.../tech docs?.../BAOSR6x11/...DIRECT\%20BI LIRU...

Smorgorzewska A, Flood JG, Long WH, Dighe AS. 2004. Paraprotein interference in autoamated clinical chemistry analyzer. Clin. Chem., 50(9): 1691-1693.

DOI:

10.1373/clinchem.2004.037499

Singh K, Rao P, Datta P, Belle VS.2015. Elevated IgG causing spurious elevation in serum total bilirubin assay. Asia Pacific J. Res., I(XXIV), Art. 21:1-5. DOI: apjor. com/downloads/160220151.pdf

Wahlefeld AW, Herz G, Bernt E. 1972. Modification of the Malloy-Evelyn method for a simple, reliable determination of total bilirubin in serum. Scand. J. Clin. Lab. Invest., 29(126): Abstract 11.12. 